\title{
NUTRIÇÃO ENTÉRICA E ALIMENTOS PARA FINS MEDICINAIS ESPECÍFICOS NA IDADE PEDIÁTRICA: REALIDADE ATUAL
}

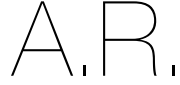
ARTIGO DE REVISÃO

1 Unidade de Nutrição Pediátrica - Centro Materno Infantil do Centro Hospitalar Universitário São João,

Alameda Prof. Hernâni

Monteiro

4200-319 Porto, Portugal

2 Serviço de Nutrição do Centro Hospitalar Universitário São João, Alameda Prof. Hernâni

Monteiro,

4200-319 Porto, Portugal

${ }^{3}$ Faculdade de Ciências de Nutrição e Alimentação da Universidade do Porto,

Rua do Campo Alegre,

n. ${ }^{\circ} 823$,

4150-180 Porto, Portugal

${ }^{4}$ Center for Health

Techonology and Services Research,

Rua Dr. Plácido da Costa,

4200-450 Porto, Portugal

5 Cooperativa de Ensino Superior Politécnico e

Universitário,

Rua Central de Gandra,

n. ${ }^{\circ} 1317$

4585-116 Gandra,

Portugal

${ }^{6}$ Faculdade de Medicina da Universidade Porto, Rua Dr. Plácido da Costa,

4200-450 Porto, Portugal

"Endereço para correspondência:

Diana e Silva

Centro Hospitalar Universitário

de São João - Serviço de

meda Prof. Hernâni Monteiro,

4200-319 Porto, Portugal

diana.mv.silva@gmail.com

Histórico do artigo:

Recebido a 17 de abril de 202 Aceite a 21 de setembro de 2021

\section{ENTERIC NUTRITION AND FOOD FOR SPECIFIC MEDICAL PURPOSES IN PEDIATRIC AGE: CURRENT REALITY}

Diana e Silva ${ }^{1-4} ;$ Marta Rola²; Ana Barreira Lopes ${ }^{3} ;$ Maria do Céu Monteiro 3,$5 ;$ Paula Guerra'; António Guerra3,4,6

RESUMO

A utilização de nutrição entérica tem um papel importante na recuperação da criança e adolescente desnutrido, ou em risco de desnutrição.

Pretende-se com a publicação deste artigo, a descrição genérica das principais questões relacionadas com a nutrição entérica onde se destacam alguns aspetos ligados ao estado de nutrição e de patologia da criança e adolescente e é feita referência às indicações, metodologia de intervenção e complicações da nutrição entérica. Estes são pontos fundamentais, para a decisão de se iniciar um programa de nutrição entérica e de se proceder à escolha da fórmula mais adequada a cada situação.

\section{PALAVRAS-CHAVE}

Idade pediátrica, Nutrição entérica

\section{ABSTRACT}

The use of enteral nutrition plays an important role in the recovery of the malnourished child or adolescent, or at risk of malnutrition. Intended with this publication, the generic description of the main issues related to enteral nutrition, where some aspects related to the state of nutrition and pathology of children and adolescents are highlighted and reference is made to the indications, methodology of enteral nutrition intervention and complications. These are important points for the decision to start an enteral nutrition program and to choose the most appropriate formula for each situation.

\section{KEYWORDS}

Pediatric age, Enteral nutrition

\section{INTRODUÇÃO}

A nutrição entérica (NE) refere-se a qualquer método de alimentação que use o trato gastrintestinal de modo a suprir em parte, ou no total, as necessidades nutricionais do doente. No sentido mais lato, e o mais amplamente aceite, o termo NE abarca as dietas fornecidas tanto pela via oral como através de uma sonda ou um estoma (1). As dietas entéricas visam, assim, alimentar de modo exclusivo, ou parcial, doentes com capacidade limitada para ingerir, digerir, absorver ou metabolizar nutrientes contidos num regime alimentar normal.

Pretende-se com esta publicação a descrição genérica das principais questões relacionadas com a NE, onde se incluem, entre outros, aspetos ligados ao estado de nutrição e de patologia da criança e adolescente. Serão abordados num segundo artigo aspetos referentes à descrição das definições, designações e classificação das fórmulas entéricas existentes no mercado até à data desta publicação. Os produtos dietéticos referidos no $2 .^{\circ}$ artigo serão classificados em função da composição estrutural dos macronutrientes, de forma a facilitar a escolha da
$\mathrm{NE}$, tendo sempre por base a idade, o quadro clinico e as necessidades nutricionais do doente.

Consideram-se estes pontos naturalmente fundamentais, para a decisão de se iniciar uma dieta entérica e de se proceder à escolha da composição mais adequada a cada situação.

\section{Utilização das Dietas Entéricas - Princípios Gerais} A Avaliação do Estado de Nutrição

A avaliação antropométrica constitui um dos procedimentos que melhor avalia o estado de nutrição em crianças e adolescentes, sendo por isso a metodologia mais utilizada na prática clínica. Para além da antropometria, a avaliação deve incluir a anamnese detalhada, incluindo os aspetos de índole socioeconómica e cultural, a ingestão qualitativa e quantitativa alimentar, o exame clínico e a avaliação bioquímica (2-4).

De acordo com a European Society for Clinical Nutrition (ESPEN), a definição de malnutrição corresponde a um termo abrangente que inclui estados de deficiência ou excesso nutricional e de desequilíbrio energético, proteico 
e/ou noutros nutrientes, causando efeitos adversos mensuráveis que podem abranger situações desde a sobrenutrição à desnutrição associadas à doença $(5,6)$.

A desnutrição é denominada de primária se resulta da escassez alimentar que origina uma ingestão alimentar diminuída e desequilibrada. Esta forma é frequente em países subdesenvolvidos, em famílias com graves carências económicas, e pode assumir formas graves de malnutrição energético-proteica como o marasmo $(5,6)$. A desnutrição subjacente a uma doença é desencadeada por uma baixa ingestão alimentar ou por um aproveitamento inadequado dos nutrientes ingeridos. Esta forma de desnutrição, classificada como secundária, decorre de doença que cursam com estados de anorexia, hipermetabolismo e de malabsorção intestinal (7).

A desnutrição tem sido associada ao aumento de mortalidade e morbilidade, à duração do internamento e ao aumento dos custos hospitalares. A desnutrição agrava o estado geral do doente, aumenta a susceptibilidade a infeções, retarda a cicatrização e produz alterações do sistema imune, cardiovascular, respiratório e digestivo, complicações estas que levam a maior tempo, e custos mais elevados, de internamento (8).

\section{A Avaliação do Risco Nutricional}

É fundamental a identificação do risco nutricional em todos os doentes, de modo a proporcionar uma intervenção precoce, evitando, ou tratando, os quadros de desnutrição e alterações metabólicas, associadas a perda de peso $(8,9)$.

É inegável que, apesar dos significativos avanços nas mais diferenciadas áreas da saúde, a prevalência de desnutrição é ainda bastante comum em crianças/adolescentes hospitalizados (8). De salientar que 20 a 50\% das crianças agravam o seu estado de desnutrição durante o período de hospitalização (6). Pelo que é mandatório iniciar precocemente um plano de intervenção nutricional adequado a cada criança/adolescente para a manutenção e/ou recuperação do estado nutricional durante o internamento hospitalar $(6,10)$.

Embora não exista consenso na definição das ferramentas mais adequadas para avaliar o risco nutricional em idade pediátrica no período de hospitalização, deseja-se que o método seja simples, rápido, de interpretação fácil, com boa sensibilidade e especificidade, além de aplicável nas múltiplas situações clínicas e por diferentes grupos profissionais de saúde. Segundo a American Society for Parenteral and Enteral Nutrition (ASPEN) e a European Society for Paediatric Gastroenterology, Hepatology, and Nutrition (ESPGHAN) a sua aplicação deve ser efetuada nas primeiras horas de internamento a todos os pacientes em situação de risco nutricional $(8,11)$. Também em Portugal foi referenciada da máxima importância a aplicação, a nível hospitalar, de um instrumento credível de quantificação do risco nutricional em idade pediátrica (12). Tal como foi descrito anteriormente a Direção Geral da Saúde (DGS) considera não haver nenhuma ferramenta "gold standard", mas reconhece com alguma credibilidade o Screening Tool for Risk of Impaired Nutritional Status and Growth (STRONGkids) para avaliação do risco nutricional em contexto de hospital hospitalar de todas as crianças com mais de 1 mês de idade e por um período de internamento superior a 24 horas, à exceção de doentes da urgência, emergência ou internados nos cuidados intensivos. Por outro lado, é de fácil utilização, não necessita de realização de medidas antropométricas e a avaliação subjetiva não demonstrou ser fator de viés (13)

\section{A Nutrição Enteral}

\section{Conceito}

O conceito de NE remonta à antiguidade, inicialmente no Egipto e mais tarde na Grécia $(14,15)$. Em 1930, pela primeira vez, os hidrolisados de proteínas foram fornecidos a doentes cirúrgicos e dez anos depois, nos anos quarenta, foi desenvolvida a primeira fórmula infantil. No entanto, foi a partir de 1980 que a NE que passou a ser reconhecida como prática segura, e economicamente viável, proporcionando uma nutrição adequada, conduzindo ao desenvolvimento de vários tipos de fórmulas com diferentes composições nutricionais destinadas a distintas situações clínicas $(15,16)$.

\section{As Indicações e as Vantagens}

Vários têm sido os estudos que advogam o uso da NE sempre que a ingestão oral esteja comprometida, já que uma atitude proativa relativa ao início do suporte nutricional por via entérica evita a perda de peso, particularmente em crianças e adolescentes internados em cuidados intensivos ou sujeitas a tratamentos muito agressivos, como quimio e radioterapia $(17,18)$.

Para além de situações em que o suprimento alimentar oral é insuficiente, a NE está indicada numa grande diversidade de situações clínicas, nomeadamente em situações associadas a problemas de digestão $e$ absorção (intestino curto, fibrose quística, doença intestinal inflamatória, entre outros), ao aumento das necessidades nutricionais (cardiopatias, fibrose quística e outras pneumopatias, doença intestinal inflamatória, grandes queimados, entre outras) ou das perdas acrescidas (fibrose quística, doença inflamatória intestinal, intestino curto, entre outras) (19). A NE é também, frequentemente usada em recém-nascidos de pretermo, por sonda nasogástrica, de forma continua ou intermitente, com o fim de ultrapassar a imaturidade digestiva e oromotora destes bébes. Um subgrupo das crianças com doenças metabólicas com intolerância ao jejum, como a doença de armazenamento do glicogénio tipo I, poderá beneficiar da nutrição entérica noturna contínua, de modo a prevenir a ocorrência da hipoglicemia. Em alguns doentes, a NE pode assumir uma função terapêutica, como é o caso das alergias ou intolerâncias alimentares e na Doença de Crohn (DC) (19, 20). Na DC, a NE exclusiva é a terapêutica de primeira linha recomendada pela ESPGHAN na indução da remissão da DC ativa na idade pediátrica $(21,22)$. Na DC, embora o mecanismo de ação da NE ainda não esteja completamente esclarecido, várias hipóteses têm sido apontadas, incluindo a modelação da microflora pelos nutrientes ou a diminuição da resposta imune através da menor exposição aos antigénios da alimentação convencional $(23,24)$. Ao que parece a NE produz um efeito anti-inflamatório direto na mucosa intestinal (24) e, nestes doentes está indicada por períodos de tempo variáveis que podem chegar até aos 12 meses de administração de uma fórmula entérica polimérica que, por definição, são fórmulas constituídas por nutrientes na sua forma intacta e requerem um intestino com normal capacidade de absorção e digestão. Estas fórmulas contêm proteínas inteiras, hidratos de carbono provenientes de oligossacarídeos, maltodextrinas ou amido, lípidos derivados de óleos vegetais, minerais, vitaminas e oligoelementos $(21,22,25)$.

Recentemente, a acompanhar o aumento do uso clínico da NE em detrimento do uso da nutrição parentérica (NP), foram lançadas no mercado português uma grande variedade de dietas entéricas com diferentes especificidades. Como vantagens apontadas para a NE relativamente à NP, salienta-se a preservação da função gastrointestinal, menor custo associado e, incomparavelmente, menores complicações infeciosas, metabólicas e hepáticas (26). A NE proporciona, a par de uma maior segurança, o fornecimento adequado de nutrientes, alguns 
específicos como é o caso da glutamina, ácidos gordos polinsaturados de cadeia longa, ácidos gordos de cadeia curta e fibras. A NE parece prevenir as alterações do fluxo pancreático e biliar associados ao jejum, bem como a disfunção endócrina pancreática (27).

Os suplementos alimentares (sip feeds), não pretendem, habitualmente, ser uma dieta completa. Independentemente da via de administração e na dependência da idade e do quadro clínico, os suplementos alimentares proporcionam um maior suprimento energético, proteico e numa variedade de nutrientes, embora alguns não forneçam todos os nutrientes de forma equilibrada $(20,28)$.

\section{Metodologia de Intervenção}

Após a avaliação do doente e do cálculo das necessidades nutricionais é escolhida a fórmula entérica mais apropriada, assim como o método e a duração da NE, tendo em conta a capacidade funcional do trato gastrointestinal $(15,19,29)$. É essencial que sempre que se opte pela NE como suporte nutricional se proceda à abordagem prévia aos pais, e das crianças mais velhas, no sentido de esclarecer a sua utilização com segurança e conquistar a confiança de todo o agregado familiar (30). Se a condição clínica do doente o permitir, a via oral e o uso das dietas poliméricas, com diferentes sabores, deve ser sempre privilegiado. Se a alimentação oral estiver contra-indicada, ou se fornecer menos de 50 \% das necessidades da criança, deverá ser instituída a nutrição entérica através de sonda nasogástrica (SNG), mais excecionalmente nasojejunal (SNJ), ou por via percutânea (gastrostomia, mais raramente jejunostomia) (31).

A NE por via gástrica é sempre preferível à jejunal. A NE por via jejunal obriga ao seu uso em débito contínuo, é menos fisiológica e está reservada para algumas situações particulares, em que via gástrica não é tolerada ou não é possível. Embora em algumas situações a alimentação por SNG possa apresentar risco de refluxo gastroesofágico e/ou aspiração pulmonar, esta, relativamente à alimentação jejunal, tem a vantagem de proporcionar um efeito bactericida importante, através do ácido clorídrico e de otimização da absorção de certos nutrientes (19).

A NE intermitente, em bolús, em comparação com a NE continua, é mais económica, mais cómoda, dispensa o uso de bomba de alimentação, consentindo uma maior liberdade ao doente na realização das suas atividades (19). A NE intermitente é mais fisiológica, permite períodos alternados de alimentação e de jejum, o que favorece um perfil mais fisiológico da produção das hormonas gastrointestinais, como a alternância dos níveis de insulina e do glucagon. Embora se trate de um método bem tolerado pela maioria dos doentes, por vezes os bolus podem desencadear episódios de diarreia osmótica (19). Neste caso, a NE contínua ou sob a forma intermitente em bolus mais lentos, pode ser uma alternativa (27).

A realização de gastrostomia/jejunostomia deve ser considerada quando se prevê que a necessidade de aporte nutricional por via entérica ultrapasse as quatro a seis semanas tanto nos adultos, como em idade pediátrica (<18 anos), após avaliação prévia das indicações, riscos e benefícios, segundo normas definidas pela DGS (31).

Estão disponíveis no mercado algumas dietas adaptadas à idade pediátrica, com macro e micronutrientes mais próximos das suas necessidades. Uma dieta padrão adequada à idade, polimérica, isoenergética ( $1 \mathrm{kcal} / 1 \mathrm{~mL}$ e 0,67 Kcal/1 mL em lactentes) e iso-osmolar (300-350 mOsm/ $\mathrm{kg}$ ) é a fórmula enteral adequada para a maioria dos pacientes pediátricos (32). Nas crianças com idades compreendidas entre 1 e 13 anos devem ser usadas as dietas indicadas para a idade pediátrica (33). Apesar de parca evidência científica, não sendo consensual na literatura, o Comité de Nutrição da ESPGHAN refere que na ausência de produtos de nutrição entérica para a idade pediátrica, poderão ser usados produtos indicados para adultos, a partir dos 8-10 anos de idade (19). Mas o uso de fórmulas de adultos em crianças mais pequenas não deve ser praticado, pelo consequente fornecimento desadequado de macro e micronutrientes, associados a uma elevada carga de solutos e de vitaminas. A diluição da fórmula entérica a fim de obter uma menor carga renal de soluto não é aconselhada pela, consequente, diluição dos micronutrientes e do compromisso suprimento nutricional oferecido, inferior às necessidades (19).

As necessidades energético-proteicas, em vitaminas e em minerais podem ser determinadas tendo por base as Dietary Reference Intake (DRI), recentemente revistas (34-38) e considerando que em determinadas patologias, alguns nutrientes devem ser suplementados ou restringidos. Estas são as mais utilizadas e as indicadas pelos Estados Unidos da América e Canadá, contudo as da Food and Agriculture Organization of the United Nations/World Health Organization (FAO/OMS) também suportaram à avaliação das recomendações alimentares da população portuguesa e industria alimentar infantil $(39,40)$. Ainda neste âmbito a European Food Safety Authority (EFSA), disponibiliza recomendações para os macronutrientes alguns micronutrientes, energia e água, elaboradas com base numa população europeia, onde se inclui Portugal e que têm vindo a sofrer atualizações sistemáticas desde 2010. A sua metodologia robusta onde se incluem recomendações de outros comités, permite com segurança serem adotadas para a população portuguesa $(41,42)$.

Do ponto de vista prático, e após o cálculo das necessidades nutricionais e da escolha da dieta mais adequada, o volume de administração inicial e a progressão, por sonda ou por gastrostomia, deve ser cautelosa de forma a permitir a adaptação do tubo digestivo e evitar a ocorrência de complicações gastrointestinais (vómitos, flatulência, distensão abdominal e a diarreia) $(43,44)$.

Assim, no início a dieta entérica deve ser ministrada com um menor volume e o seu ritmo aumentado de acordo com a tolerância digestiva. O ritmo, e eventualmente a concentração da fórmula, são progressivamente aumentados até ao máximo estabelecido. O aumento simultâneo da concentração e do volume pode ser mal tolerado, pelo que habitualmente se procede a aumentos graduais de volume com a fórmula escolhida, habitualmente isoenergética (1 a 1,2 Kcal/mL), e, depois se necessário, aumenta-se a concentração da fórmula.

Na Tabela 1 figuram algumas orientações práticas para o início e progressão da nutrição entérica $(43,44)$.

\section{As Contra-indicações}

São contraindicações absolutas para NE sempre que se observem alterações graves da função intestinal tais como: obstrução intestinal (mecânica ou funcional); perfuração intestinal, peritonite e a enterocolite necrotizante. São consideradas contraindicações relativas a dismotilidade intestinal, o megacólon tóxico, o sangramento gastrointestinal ou fístula ativa. Contudo, sempre que possível o jejum completo deve ser evitado, mantendo-se o fornecimento mínimo de nutrientes, de modo a promover a função intestinal (libertação de hormonas enterais) e a preservar a integridade da barreira intestinal $(28,49)$.

\section{Complicações}

Existem algumas complicações relacionadas com a NE, associadas a sonda (SNG, SNJ), à presença de estoma e à dieta entérica (tipo de dieta, ritmo e modo de infusão). A presença de uma sonda, para além de desconforto nasofaríngeo, tem o risco de obstrução ou de 
Tabela 1

Ritmos e volumes de administração da nutrição entérica

\begin{tabular}{|c|c|c|c|}
\hline IDADE & VOLUME INICIAL & PROGRESSÃO & VOLUME FINAL \\
\hline \multicolumn{4}{|c|}{ Nutrição Entérica Contínua } \\
\hline $0-12$ meses & $1-2 \mathrm{~mL} / \mathrm{kg} / \mathrm{h}$ & $1-2 \mathrm{~mL} / \mathrm{kg} / \mathrm{h}$ (a cada $2-8 \mathrm{~h})$ & $5-7 \mathrm{~mL} / \mathrm{kg} / \mathrm{h}$ \\
\hline $1-6$ anos & $2-3 \mathrm{~mL} / \mathrm{kg} / \mathrm{h}$ & $1 \mathrm{~mL} / \mathrm{kg} / \mathrm{h}$ (a cada 2-8h) & $4-5 \mathrm{~mL} / \mathrm{kg} / \mathrm{h}$ \\
\hline $7-14$ anos & $1 \mathrm{~mL} / \mathrm{kg} / \mathrm{h}$ & $0,5 \mathrm{~mL} / \mathrm{kg} / \mathrm{h}$ (a cada $2-8 \mathrm{~h})$ & $3-4 \mathrm{~mL} / \mathrm{kg} / / \mathrm{h}$ \\
\hline$\geq 14$ anos & $0,5-1 \mathrm{~mL} / \mathrm{kg} / \mathrm{h}$ & $0,4-0,5 \mathrm{~mL} / \mathrm{kg} / \mathrm{h}$ (a cada $2-8 \mathrm{~h}$ ) & $125 \mathrm{~mL} / \mathrm{h}$ \\
\hline \multicolumn{4}{|c|}{ Nutrição Entérica Intermitente (contraindicada na via jejunal) } \\
\hline $0-12$ meses & $10-15 \mathrm{~mL} / \mathrm{kg} / \mathrm{toma}$ & $10-30 \mathrm{~mL} /$ toma & $20-30 \mathrm{~mL} / \mathrm{kg} / \mathrm{toma}$ \\
\hline $1-6$ anos & $5-10 \mathrm{~mL} / \mathrm{kg} / \mathrm{toma}$ & $30-45 \mathrm{~mL} /$ toma & $15-20 \mathrm{~mL} / \mathrm{kg} / \mathrm{toma}$ \\
\hline $7-14$ anos & 3-5 mL/kg/toma & $60-90 \mathrm{~mL} /$ toma & $10-20 \mathrm{~mL} / \mathrm{kg} / \mathrm{toma}$ \\
\hline$\geq 14$ anos & $3 \mathrm{~mL} / \mathrm{kg} / \mathrm{toma}$ & $100 \mathrm{~mL} /$ toma & $300-500 \mathrm{~mL} /$ toma \\
\hline
\end{tabular}

Adaptada de J Álvarez et al., 2011 (45); R. A. Lama More et al., 2015 (46); Moreno JM et al., 2005 (47); Davis AM, 2007 (48)

deslocação. A gastrostomia percutânea endoscópica (cujas vantagens e desvantagens devem ser cuidadosa e previamente avaliadas) têm também algumas complicações associadas, particularmente as relativas à pele da zona peri-estoma (irritação cutânea, dor, sangramento, celulite e abcesso peristomal), à dimensão do estoma (saída de nutrientes ou de suco gástrico) e, mais raramente, sépsis com ponto de partida na infeção do estoma $(15,19,50,51)$.

As complicações infeciosas podem, também, ter origem na contaminação das fórmulas e no não cumprimento dos procedimentos de manipulação e armazenamento (52). Por vezes, têm sido reportadas aos hospitais pediátricos ocorrências de contaminação bacteriana e situações de sépsis, mas cujo relato nem sempre é muito preciso (53). As complicações gastrointestinais incluem desconforto abdominal, distensão abdominal e cólicas (15). Náuseas e vómitos associam-se a elevados volumes de infusão, ao esvaziamento gástrico lento, obstipação e a fatores psicológicos. Para além disso, a deslocação dos tubos e intolerância aos bolus alimentares podem conduzir a regurgitação e até aspiração (19).

As complicações metabólicas durante a NE podem estar presentes em doentes com desequilíbrio nutricional crónico ou com distúrbios cardíacos, hepáticos ou renais. Os doentes muito desnutridos quando são realimentados correm o risco de desenvolver, ou de agravar, desequilíbrios metabólicos, nomeadamente a síndrome de realimentação, pelo que a administração abrupta de NE hiperenergética deve ser evitada (54). Nesta situação, em resposta ao fornecimento de quantidades elevadas de hidratos de carbono, ocorre um aumento da secreção de insulina o que leva a uma deslocação para o interior das células de iões de fósforo, magnésio e potássio diminuindo as suas concentrações séricas. Em caso de ocorrência de hipofosfatemia, esta pode levar a insuficiência cardíaca, arritmias e morte. De forma a evitar o desenvolvimento do síndrome de realimentação, o fornecimento do volume e valor energético alimentar deve ser inferior a $75 \%$ das necessidades, aumentando-se gradualmente até atingir as necessidades calculadas (27).

Como complicação da NE, pode, ainda, ocorrer uma inesperada interação fármaco-nutriente quando os medicamentos são administrados por tubo de alimentação enteral $(19,15)$. Mas caso não exista outra alternativa de fornecimento dos medicamentos, e de forma a minimizar as complicações como a precipitação dos fármacos, obstrução das sondas ou sua aderência às paredes das sondas, estes devem ser ministrados em pequenas porções, os comprimidos podem ser desfeitos em água e as cápsulas de gelatina dissolvidas em água morna (27).

\section{CONCLUSÕES}

A NE refere-se a qualquer método de alimentação que use o trato gastrintestinal (por via oral ou através de sondas ou de estomas) de modo a suprir em parte ou no total as necessidades nutricionais do doente, sendo segura e eficaz.

A NE está indicada quando a alimentação oral é insuficiente para assegurar o crescimento normal e quando a função gastrointestinal está total, ou parcialmente, preservada.

A formulação da terapia nutricional entérica deve ser ajustada às necessidades específicas do doente e da doença. Na maior parte dos doentes, as necessidades energéticas podem ser satisfeitas com fórmulas com densidade calórica de $1 \mathrm{kcal} / \mathrm{mL}$. Nas crianças com idades compreendidas entre 1 e 13 anos devem ser usadas as dietas indicadas para a idade pediátrica.

Sempre que possível é desejável a opção pela via gástrica direta, em vez de fornecimento de nutrientes por sonda trans-pilórica A NE por via gástrica é mais fisiológica e permite a alimentação intermitente, em bolus, ao invés da via jejunal que obriga a alimentação por débito contínuo. Quando se prevê que a necessidade de aporte nutricional por via entérica ultrapasse as 4 a 6 semanas a via percutânea, gastrostomia, e muito mais raramente jejunostomia, deve ser equacionada.

As complicações técnicas, metabólicas, gastrointestinais, infeciosas e psicológicas podem ser minimizadas através da monitorização regular do protocolo e da avaliação clínica de cada doente.

O presente trabalho é complementado pela parte II onde é feita uma revisão exaustiva das dietas entéricas disponíveis atualmente no mercado Português.

\section{REFERÊNCIAS BIBLIOGRÁFICAS}

1. Lochs $\mathrm{H}$, Allison SP, Meier R, et al. Introduction to the ESPEN Guidelines on Enteral Nutrition. Terminology, definitions and general topics. Clin Nutr 2006;25:180-6. 2. World Health Organization. WHO Child Growth standarts-training course on child Growth Assessment Geneva: WHO, 2008.

3. CDC Monotoring the Nation's Health. Antropometric Reference Data for Children and Adults: United States, 2007 - 20110. U.S. Department of Health and Human Services. 2012.

4. Nagy P, Kovacas E. Percentile reference values for anthropometric body composition indices in European children from the IDEFICS study. International Journal of Obesity. 2014;38: S15 - S25.

5. Arends J, Bachmann P, Baracos V, Barthelemy N, Bertz H, Bozzetti F, Fearon K, Hutterer E, Isenring E, Kaasa S,Krznaric Z et al. ESPEN guidelines on nutrition in cancer patients. Clinical Nutrition 2016; XXX: 1 - 38.

6. Hartman C, Shamir R, Hecht C, Koletzko B. Malnutrition screening tools for hospitalized children. Curr Opin Clin NUTR Metab Care . 2012;15(3): 303-309. 
7. Aurangzeb B, Whitten K, Harrison B, Mitchell M, Kepreotes H, Sidler M, Lemberg $D$, Day A. Prevalence of malnutrition and risk of under-nutritionin hospitalized children. Clin Nutr. 2012, 31(1):35-40.

8. Joosten KF, Hulst JM. Nutritional screening tools for hospitalized children: methodological considerations. Clin Nutr. 2014; 33(1):1-5.

9. Becker PJ et al. consensus statement of the Academy of Nutrition and Dietetics American Society for Parenteral and Enteral Nutrition: indicators recommended for the identification and documentation of pediatric malnutrition (undernutrition)). Journal of the Academy of Nutrition and Dietetics. 2014; 114(12):1988-200.

10. Guerra R, Fonseca I, Pichel F, Restivo M, Amaral T. Usefulness of six diagnostic and screening measures for undernutrition in predicting length of hospital stay: $A$ comparative analysis. J Acad Nutr Diet; 2015; 115 (6): 927-938.

11. Chourdakis M, Hecht C, Gerasimidis K, Joosten KF, Karagiozogiozoglou-Lampoudi, Ksiazyk J, Lazea C, Shamir R, Szajewska H, Koletzko B, Hulst JM. Malnutrition risk in hospitalized children: use of 3 screening tools in a large European population. Am J Clin Nutr. 2016; 103 (5):1301-10

12. Matos C, Faria A, Vasconcelos C, Tomada I, Grós Dias MP, Moreira Identificação do Risco Nutricional em Idade Pediátrica. Norma de Orientação Profissional. NOP 001/2018; Versão 01: 2-16.

13. Huysentruyt K, Allie P Fau- Muyshont, Muyshont Fau- Rossignol R, Rossignol R Fau - Devreker T Devreker T, Devreker TFau - Bontems P, Bontems P Fau - Dejonckheere J, et al. The STRONG(kids) nutritional screening tool in hospitalized children: a validation study. Nutrition. 2013; 29(11-12):1356-61.

14. Harkness $L$. The history of enteral nutrition therapy: from raw egges and nasal tubes to purified amino acids and early postoperative jejuna delivery. J. Am Diet Assoc 2002; 102:399-404

15. McCallum Z, Biness JE. Enteral Nutrition and Formulas. In Duggan C, Watking JB, Walker WA, eds. Nutrition in pediatrics. 5th ed Shelton, CT:PMPH-USA,2016:1023-34 16. Philips MS, Ponsky JL. Overview of enteral and parenteral feeding acess techniques: principles and practice. Surg Clin North Am 2011:91:897-911.

17. Sacks N, Hwang WT, Lange BJ, Tan KS, Sandler ES, Rogers PC, et al. Proactive enteral tube feeding in pediatric patients undergoing chemotherapy. Pediatric blood \& cancer. 2014; 61 (2): 281-5

18. Bagonzi E, Buteyn M, Lagana E. Best Practices in Pediatric Oncology: Enteral Versus Parenteral Nutrition. Journal of the Academy of Nutrition and Dietetics. 2015;113(9):A27 (25).

19. Braegger C, Decsi T, Amil Dias J, Hartman C, Kolacek S, Koletzko B, Koletzko S, Mihatsch W, Moreno L, et al. Practical Approach to Paediatric Enteral Nutrition: A Comment by the ESPGHAN Committee on Nutrition. Journal of Pediatric Gastroenterology and Nutrition. 2010; 51: 110-122.

20. Commission Directive 1999/21/EC of 25 March 1999 on dietary foods for special medical purposes. http://www.idace.org/ legislation/fsmps/Dir\%2099-21\%20FSMPs. pdf) 21. Frivolt K, Schwerd T, Werkstetter KJ, Schwarzer A, Schatz SB, Bufler P, et al. Repeated exclusive enteral nutrition in the treatment of paediatric Crohn's disease: predictors of efficacy and outcome. Alimentary Pharmacology \&Therapeutics. 2014;39(12):1398-407.

22. Soo J, Malik BA, Turner JM, Persad R, Wine E, Siminoski K, et al. Use of exclusive enteral nutrition is just as effective as corticosteroids in newly diagnosed pediatric Crohn's disease. Digestive Disease. Digestive Diseases and Sciences. 2013;58(12):3584-91

23. Altomare R, Damiano G, Abruzzo A, Palumbo VD, Tomasello G, Buscemi S, et al. Enteral nutrition support to treat malnutrition in inflammatory bowel disease. Nutrients. 2015;7(4):2125-33.

24. Critch J, Day AS, Otley A, King-Moore C, Teitelbaum JE, Shashidhar H. Use of enteral nutrition for the control of intestinal inflammation in pediatric Crohn disease. Journal of Pediatric Gastroenterology and Nutrition. 2012; 54(2):298-305.

25. Zádak Z, Kent-Smith L. Basics in clinical nutrition: Commercially prepared formulas. The European e-Journal of Clinical Nutrition and Metabolism. 2009; 4: e212-e215. 26. Gramilch L, KICHIAN k, Pinilla J, Rodych NJ, Dhaliwal R, Heyland DK. Does enteral nutrition compared to parenteral nutrition result in better outcomes in critically ill adult patients? A systematic review of the literature. Nutrition 2004; 20:843-8. 27. Dae Yong Yi. Enteral Nutrition in Pediatric Patients. Pediatr Gastroenteral Hepatol Nutr. 2018; 21(1):12-19

28. Tyson JE, Kennedy KA. Minimal enteral nutrition for promoting feeding tolerance and preventing morbidity in parenterally fed infants. Cochrane Database Syst Rev 2000;(2):CD000504.

29. Avitzur Y, Courtney-Martin G. Enteral approaches in malabsorption. Best Pract Res Clin Gastroenterol 2016;30:295-307.

30. Montgomery K, Belongia M, Haddigan M M, Schulta C, Philips S, Simpson PM et al. Perceptions of nutrition support in pediatric oncology patients and parents. Journal of Pediatric Oncology Nursing: official journal of the Association of Pediatric Oncology Nurses. 2013; 30(29):90-8.

31. DGS. Norma $n^{\circ}$ 014/2016 de 28/10/2016 atualizada a 03/03/2017. Indicações Clínicas e Intervenção nas Ostomias de Alimentação em Idade Pediátrica e no Adulto. https://www.dgs.pt/directrizes-da-dgs/normas-e-circulares-normativas/norma-n-0142016-de-28102016-pdf.aspx.

32. Koletzko B, et al. (eds): Pediatric Nutrition in Practice. World Rev Nutr Diet. Basel, Karger, 2015, vol 113, pp 152-157.

33. Enteral Nutrition. In: Kleiman RE, Geer FR (eds). Pediatric Nutrition (7th ed). American Academy of Pediatrics. New York, 2013: 591-605.

34. Food and Nutrition Board, Institute of Medicine. Dietary Reference Intakes: Applications in Dietary Planning. Washington, DC: National Academies Press, 2003.

35. Food and Nutrition Board, Institute of Medicine. Dietary Reference Intakes for Calcium, phosphorus, magnesium vitamin D, and fluoride. Washington, DC: National Academies Press, 2003.

36. Food and Nutrition Board, Institute of Medicine. Dietary Reference Intakes for vitamin C, vitamin E, selenium and carotenoides. Washington, DC: National Academies Press, 2000.

37. Food and Nutrition Board, Institute of Medicine. Dietary Reference Intakes for energy, carbohydrates, fiber, fat, fatty acids, cholesterol, proteins and aminoacids. Washington, DC: National Academies Press, 2006.

38. Food and Nutrition Board, Institute of Medicine. Dietary Reference Intakes for Calcium and vitamin D. Washington, DC: National Academies Press, 2011.

39. Valente H, Padez C, Mourão I, Rosado V, Moreira P, Prevalencia de Inadquação nutricional em crianças portuguesas. Acta Med Port, 2010; 23: 365-370.

40. FAO/WHO (Food and Agriculture Organization of the United Nations/World Health Organization). FAO/WHO Technical Consultation on National Food - based Dietary Guidelines Reporto f Joint FAO/WHO Expert Consultation, Cairo , Egypt 6-9 December 2004; 2006.

41. EFSA Paniel on Dietetic Products Nutrition, and Alergies (NDA). Scientific Opinion on principlesfor deriving and applying Dietary Reference Values.. 2010;8(3):1458. 42. Nazareth M, Rêgo C, Lopes C, Pinto E. Recomendações nutricionais em idade pediátrica: o estado da arte. Acta Portuguesa de Nutrição. 2016 (07): 18-33.

43. Baker S. Enteral nutrition in pediatrics. In: Rombeau JL, Rolandelli RH (eds). Clinical nutrition. Enteral and tube feeding. Philadelphia: W.B. Saunders Company, 1997;349-367.

44. López Ruzafa E, Galera Martínez R, Cortés Mora P. Elección de la fórmula. En: Manual de nutrición enteral en Pediatría. Ed. Rosa A. Lama More. Grupo GETNI. Ed Glosa. Barcelona; 2015

45. J. Álvarez, G. Olveira, M. ${ }^{a}$ M. Cervera Peris, A. Mesejo, Á. Gil Hernández, J. Culebras, E. Camarero Documento de consenso SENPE/SEGHNP/ANECIPN/SECP sobre vías de acceso en nutrición enteral pediátrica. Nutr Hosp. 2011;26(1):1-15.

46. R.A. Lama More, R. Galera Martínez. Nutrición enteral. Pediatr Integral 2015; XIX (5): 365.e1-365.e6.

47. Moreno JM, Pedrón C. Nutrición enteral en el paciente pediátrico. En: Gil A, ed. Tratado de Nutrición. Madrid: Acción Médica; 2005; pp. 235-66.

48. Davis AM. Transitional and combination feeding. En: Baker SS, Baker RD, Davis AM, eds. Pediatric nutrition support. Sudbury: Jones and Barlett publishers; 2007; pp. 261-72.

49. Ohta K, Omura K, Hirano K, Kanehira E, Ishikawa N, Kato Y, et al. The effects of an 
additive small amount of a low residual diet against total parenteral nutrition-induced gut mucosal barrier. Am J Surg 2003;185:79-85.

50. Dautle MP, Wilkinson TR, Gauderer MW. Isolation and identification of biofilm microorganisms from silicone gastrostomy devices. J Pediatr Surg 2003;38:216-20. 51. Avitsland TL, Kristensen C, Emblem R, Veenstra M, Mala T, Bjørnland K. Percutaneous endoscopic gastrostomy in children: a safe technique with major symptom relief and high parental satisfaction. J Pediatr Gastroenterol Nutr 2006;43:624-8.

52. Bott L, Husson MO, Guimber D, Michaud L, ArnaudBattandier F, Turck D, et al. Contamination of gastrostomy feeding systems in children in a home-based enteral nutrition program. J Pediatr Gastroenterol Nutr 2001;33:266-70.

53. Roy S, Rigal M, Doit C, Fontan JE, Machinot S, Bingen E, et al. Bacterial contamination of enteral nutrition in a paediatric hospital. J Hosp Infect 2005;59:311-6. 54. Kraft MD, Btaiche IF, Sacks GS. Review of the refeeding syndrome. Nutr Clin Pract 2005;20:625-33. 